\title{
An effective excess charge model to describe hysteresis effects on streaming potential
}

\author{
M. Soldi ${ }^{1 *}$, L. Guarracino ${ }^{1}$ and D. Jougnot ${ }^{2}$ \\ ${ }^{1}$ Facultad de Ciencias Astronómicas y Geofísicas, Universidad Nacional de La Plata, Consejo Nacional \\ de Investigaciones Científicas y Técnicas, La Plata, Argentina \\ ${ }^{2}$ Sorbonne Université, CNRS, EPHE, UMR 7619 METIS, Paris, France \\ *E-mail: msoldi@fcaglp.unlp.edu.ar
}

This paper has been published in Journal of Hydrology:

Soldi, M., Guarracino, L. and Jougnot, D. An effective excess charge model to describe hysteresis effects on streaming potential. Journal of Hydrology (2020).

https://doi.org/10.1016/j.jhydrol.2020.124949

\begin{abstract}
Streaming potentials are produced by the coupling between the water flow and the electrical current generated by the drag of electrical charges within the pore water of the media. This electrokinetic coupling is strongly influenced by the hydraulic properties that control groundwater flow (permeability, saturation and pressure head). Under unsaturated conditions, hydrogeologic studies have widely established that the relationships of permeability and saturation with pressure head are different for drainage and imbibition experiments. The hysteresis phenomenon present on these properties produces a hysteretic behaviour on the streaming potential which has been recently observed in experimental data. Hysteresis can be explained by the presence of irregularities in the pore geometry of the media which affects the water flow and, therefore, the excess charge density that is effectively dragged by the flow. In this study, we present a physically-based analytical model to describe the hysteresis phenomenon in the estimates of the effective excess charge density. Under the assumptions of a porous medium represented by a bundle of tortuous capillary tubes with throats and a fractal pore size distribution, hysteretic curves are obtained for the effective excess charge density as a function of pressure head using a flux averaging technique. These analytical expressions are closed-form and depend on the medium petrophysical and chemical properties. The predictions of the proposed model are consistent with laboratory data from drainage-imbibition experiments. These results open up exciting possibilities for studies involving water movement and processes in the vadose zone.
\end{abstract}

Keywords: Hydrogeophysics, Streaming Potential, Hysteresis, Vadose zone

\section{Introduction}

Understanding and monitoring water movement in the subsurface is important to characterize the processes occurring in the earth critical zone. Indeed, water plays crucial roles 
in supporting terrestrial life, shaping and interacting with that zone (Fan et al., 2019). Among the different electrical methods used to study groundwater, the self-potential (SP) method has proven to be the most appropriate for characterizing water flow since its sensitivity to water flux direction and velocity. This passive geophysical method relies on the measurement of electrical potential differences (i.e., the electrical field) using two or more non-polarizable electrodes (e.g., Petiau, 2000) and a high impedance voltmeter. It can be employed performing snapshots or monitoring of profiles, maps (e.g., Jardani et al., 2006), or vertically distributed in the ground (e.g., Doussan et al., 2002; Jougnot et al., 2015). The SP method was effectively used to monitor pumping and recovery tests (e.g., Rizzo et al., 2004; Straface et al., 2007; Malama et al., 2009b; Soueid Ahmed et al., 2014), and also environmental studies such as $\mathrm{CO}_{2}$ flooding (e.g., Büsing et al., 2017), contaminant fluxes (e.g., Linde and Revil, 2007) and root-water uptake (e.g., Voytek et al., 2019). The recorded SP signals are a superposition of different contributions related to electrokinetic, redox and diffusion phenomena. In this study, we focus on the electrokinetic (EK) contribution which is predominant in hydrological studies as it is generated from the water flow in porous media. The origin of the EK contribution to the SP signal lies in the presence of an electrical double layer (EDL) in the pore water created by the electrical charges that are generally found at the mineral surface (Stern, 1924). The EDL contains an excess of charge that counterbalances the charge deficiency of the mineral surface. This excess of charge is distributed in two layers within the EDL (see Fig. 1). Close to the mineral surface is where most of the excess charge is distributed in a fixed layer with a very limited thickness called the Stern layer, and the rest of the remaining fraction is contained and flows in the diffuse layer (or Gouy-Chapman layer). The limit between these two layers can be approximated by the shear plane which is characterized by an electrical potential called $\zeta$-potential (e.g. Hunter, 1981).

Flow and transport in partially saturated soils are significantly influenced by the hysteresis phenomenon present in the hydraulic properties of the porous media (e.g., Topp, 1971; Mualem, 1977; Jury et al., 1991; Pham et al., 2005). Recent studies have shown the importance of the hysteresis phenomenon regarding the SP signal. Doussan et al. (2002) measured SP signals during rainfall events and observed differences in the water flux estimates when considering drainage and imbibition phases. Maineult et al. (2008) measured variations of the SP signal during periodic pumping tests performed at a test site located near a freshwater reservoir. Whereas they observed a correlation between the pumping and the SP signal, a phase-lag was found between the SP and pressure head measurements which they related to drainage-imbibition cycles. Revil et al. (2008) performed numerical experiments with harmonic pumping tests in an unconfined aquifer. They observed that the experiment accounting for a hysteretic flow model could explain the SP variations found by Maineult et al. (2008). In order to extract valuable information from pumping tests using SP data, it is necessary to rely on accurate models. For example, Malama et al. (2009a) developed mathematical solutions for the SP signals associated to pumping test in unconfined aquifers. Later, Soueid Ahmed et al. (2016) developed a hydraulic tomography approach for an aquifer in transient conditions from SP and hydraulic head data. In this work the authors mention the importance of the hysteresis of the hydraulic 
properties in the SP signals. Haas and Revil (2009) measured SP signals resulting from Haines jumps during the drainage and imbibition of a sandbox and observed that in each case the electrical signature was different. The drainage experiment exhibited a larger amount of electrical bursts in the SP signals than the imbibition experiment. Jougnot et al. (2012) developed two flux averaging approaches to estimate the EK contribution to the SP signal by considering that the pore distribution of the media can be derived from the water retention function or from the relative permeability function. They tested both approaches against an unsaturated vertical hydraulic flux due to rainfall events from Doussan et al. (2002). While their model predicted well the first rainfall, the following events presented an increasing discrepancy. They considered that hysteretic effects due to drainage-imbibition cycles of the soil may explain that observation. Allègre et al. (2014) performed a study of the SP response to drainage and imbibition experiments in a sand column and observed that the SP signal presented a hysteretic behaviour with respect to pressure head. Later, Zhang et al. (2017) presented a methodology to determine a relationship between the streaming potential coupling coefficient and saturation for unsaturated flow during drainage and imbibition experiments.

In order to study SP phenomena, two approaches have been developed over the years to model the streaming current generation. On the one side, the Helmholtz-Smoluchowski coupling coefficient approach focuses on the evolution with varying water saturation of the coupling coefficient which relates an electrical potential and a hydraulic pressure head differences (Guichet et al., 2003; Jackson, 2010; Allègre et al., 2014). This approach neglects electrical surface conductivity on the mineral surface, nevertheless alternative formulas have been proposed by several researchers in order to account this effect (e.g., Morgan et al., 1989; Revil et al., 1999; Glover and Déry, 2010). On the other side, the second approach is more recent and focuses on the excess charge that is effectively dragged by the water flux in the pore space (e.g., Kormiltsev et al., 1998; Linde et al., 2007; Revil et al., 2007; Jougnot et al., 2012; Revil, 2017; Guarracino and Jougnot, 2018; Jougnot et al., 2020). In this approach, the streaming current can be expressed as the product between the effective excess charge density and the water flux velocity. While both approaches describe the same physics, the difference lies in which parameter is used to describe the electrokinetic coupling between the streaming potential and the water flux. An interesting point of the second approach is that it allows the decomposition of the coupling coefficient in three components: the relative permeability, the electrical conductivity and the effective excess charge density. Whereas all of these components depend on the water saturation, the behaviour of each one is different. The behaviour of the first two components under unsaturated conditions has been studied for decades (e.g., Archie et al., 1942; Waxman and Smits, 1968; Mualem, 1986; Lenhard and Parker, 1987), nevertheless, how the effective excess charge density varies with saturation is a current theme of study that requires more development (e.g., Jougnot et al., 2012; Revil, 2017; Thanh et al., 2018; Soldi et al., 2019).

Based on the coupling coefficient approach, Revil et al. (2007) proposed the first model to describe the hysteresis phenomenon in the streaming potential. They considered two sets of van Genuchten parameters (one for the drainage and one for the imbibition 
experiments) in order to model this phenomenon in the variation of the coupling coefficient with the saturation. Later, Jougnot and Linde (2013) also employed this approach to reproduce the SP signal during drainage and imbibition experiments. In this study, we consider the framework developed by Sill (1983) and focus on the effective excess charge approach proposed by Kormiltsev et al. (1998) and Revil et al. (2007) where the EK signal can be directly related to the water flux velocity:

$$
\nabla \cdot(\sigma \nabla \varphi)=\nabla \cdot\left(\hat{Q}_{v} \mathbf{u}\right)
$$

being $\sigma\left(\mathrm{S} \mathrm{m}^{-1}\right)$ the bulk electrical conductivity, $\varphi(\mathrm{V})$ the electrical potential, $\hat{Q}_{v}(\mathrm{C}$ $\left.\mathrm{m}^{-3}\right)$ the excess charge effectively dragged by the water flux and $\mathbf{u}\left(\mathrm{m} \mathrm{s}^{-1}\right)$ the water flux which follows Buckingham-Darcy's law (Buckingham, 1907; Darcy, 1856).

In order to describe the water flow at the representative elementary volume (REV) scale, capillary tube models have proven to be useful for characterizing the porous media by considering different shapes and pore size distributions (e.g., Jerauld and Salter, 1990; Xu and Torres-Verdín, 2013; Wang et al., 2015). Recently, these models have provided valuable insight in the study of, for example, mineral dissolution (e.g., Guarracino et al., 2014), electrical conductivity (e.g., Thanh et al., 2019), saturation hysteretic effects on seismic signatures (e.g., Solazzi et al., 2019) and streaming potential phenomenon (e.g., Jackson, 2008, 2010; Linde, 2009; Jougnot et al., 2012, 2015; Thanh et al., 2018; Guarracino and Jougnot, 2018). In this study, we derive an analytical model to describe the hysteresis phenomenon in the effective excess charge density under partially saturated conditions. For this purpose, we base our approach on the capillary tube model proposed by Soldi et al. (2017) that only describes the hydraulic properties of a partially saturated porous media. The key feature of this model is that it includes hysteresis effects in the water flow properties by considering irregularities in the structure of the tubes. The pore geometry of this model is represented by a bundle of capillary tubes with periodic reductions in the pore radius (constrictivities or "ink-bottle") and a fractal pore size distribution. This pore geometry causes a different saturation pattern during drainage and imbibition that can be used to model hysteresis in macroscopic hydraulic properties. Nonetheless, other effects could also contribute or explain the presence of the hysteresis phenomenon in porous media such as contact angle effects, entrapped air and pore network connectivity (e.g., Jury et al., 1991; Klausner, 1991; Vogel and Roth, 2001). Therefore, assuming this pore geometry, the excess charge effectively dragged by the water flow is first calculated for one single constrictive capillary tube (i.e. referred to as microscale) and is then upscaled to the bundle of capillary tubes (i.e. the REV scale) using a flux-averaging technique. Closed-form analytical expressions for the effective excess charge density are obtained as a function of pressure head. The periodic constrictivities of the pores allow to introduce the hysteresis phenomenon in the model's expressions in a simple form due to the strong control of those irregularities over the water flow. The proposed model is consistent with the previous model of Soldi et al. (2019) for non-constrictive capillary tubes, and with experimental laboratory data from drainage and imbibition cycles. Moreover, the relationship between the effective excess charge density and the coupling coefficient allowed us to estimate this last coefficient for different soil textures and also observed its 
hysteretic behaviour when expressed as a function of pressure head.

\section{Hysteretic analytical model}

In the present section, we derive an analytical closed form expression for the effective excess charge density. The proposed model is based on the macroscopic description of the effective excess charge density that is dragged by the water flow in the porous media and that can be obtained from the upscaling of pore size flow and electrokinetic phenomena. First, we present the pore geometry, the pore size distribution law and the hysteretic hydraulic properties obtained at macroscopic scale from Soldi et al. (2017). Then, we derive the electrokinetic properties, for a single pore and for a REV of porous media, and we obtain effective excess charge density curves for drainage and imbibition as functions of pressure head.

At microscopic scale, we consider that the pore structure of the media is represented by tortuous capillary tubes with varying aperture. Each pore is conceptualized as a circular tube of radius $R(\mathrm{~m})$ and length $l(\mathrm{~m})$ with periodically pore throats $a R$ (as illustrated in Fig. 1) where $a$ is the radial factor that represents the ratio in which the radius is reduced ( $0 \leq a \leq 1$, dimensionless). Then, under the assumption that the pore geometry has a wavelength $\lambda$ and that the length of the tube contains an integer number $M$ of wavelengths, the pore radius along the tube can be expressed as (Soldi et al., 2017):

$$
r(x)=\left\{\begin{array}{lll}
a R & \text { if } & x \in[0+\lambda n, \lambda c+\lambda n) \\
R & \text { if } & x \in[\lambda c+\lambda n, \lambda+\lambda n),
\end{array}\right.
$$

where $c$ is the length factor $(0 \leq c \leq 1$, dimensionless) that represents the segment of $\lambda$ with pore throat and $n=0,1, \ldots, M-1$.

At macroscopic scale, we consider as a representative elementary volume (REV) a cylinder of radius $R_{R E V}(\mathrm{~m})$ and length $L(\mathrm{~m})$. The porous space of the REV is represented by a bundle of capillary tubes whose radii vary between a minimum $R_{\min }(\mathrm{m})$ and a maximum $R_{\max }(\mathrm{m})$ pore radius value.

The number of pores whose radii are greater than or equal to $R$ is assumed to follow a fractal law given by (Tyler and Wheatcraft, 1990; Yu et al., 2003; Soldi et al., 2017, 2019):

$$
N(R)=\left(\frac{R_{R E V}}{R}\right)^{D},
$$

where $D$ is the pore fractal dimension $\left(1<D<2\right.$, dimensionless) and $0<R_{\text {min }} \leq R \leq$ $R_{\max }<R_{R E V}$.

Differentiating the cumulative pore size distribution given by Eq. (3) with respect to $-R$, we obtain the number of pores whose radii are within the infinitesimal range $R$ and $R+d R:$

$$
d N(R)=D R_{R E V}^{D} R^{-D-1} d R .
$$




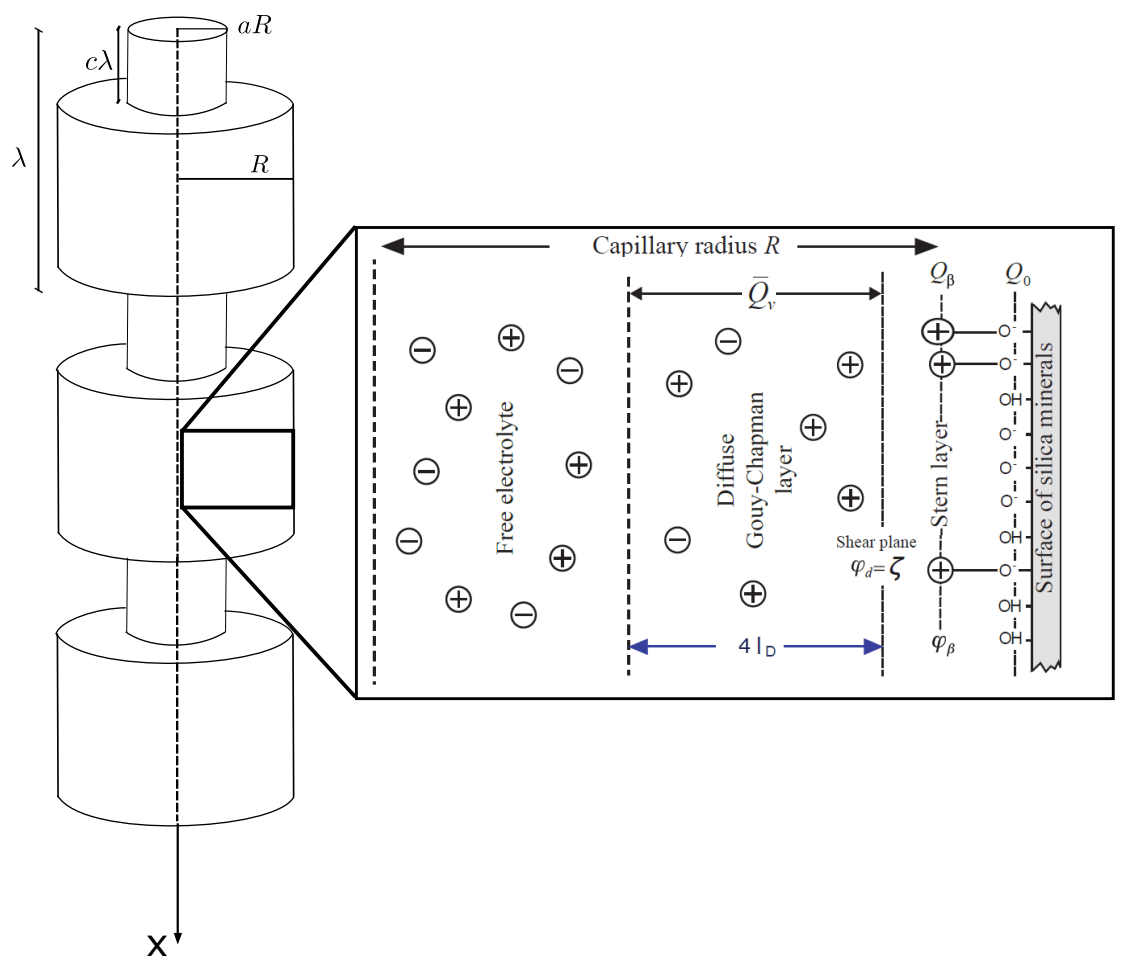

Figure 1: Schemes of the pore geometry of a single capillary tube with periodic pore throats (on the left) and of the electrical layers within the capillary (on the right). 


\subsection{Hydraulic properties}

In this Section, we present the expressions of the REV's porosity, and the effective saturation and relative permeability curves as function of the pressure head from Soldi et al. (2017). These curves are different for drainage and imbibition tests due to the presence of the pore throats.

The porosity $\phi$ of the REV can be computed from its definition as the ratio of the pore volume to the total volume of the REV. For the proposed geometry, the expression of $\phi$ yields:

$$
\phi=\frac{f_{v}(a, c) D \tau}{R_{R E V}^{(2-D)}(2-D)}\left[R_{\max }^{2-D}-R_{\min }^{2-D}\right],
$$

where $\tau=l / L$ (dimensionless) is the hydraulic tortuosity of the pores and

$$
f_{v}(a, c)=a^{2} c+1-c .
$$

This factor $f_{v}$ varies between 0 and 1 (see Fig. 2a from Soldi et al., 2017) and quantifies the reduction in the pore volume due to the presence of the pore throats.

To obtain the main drying effective saturation curve, we consider that the REV is initially fully saturated and a pressure head $h$ is applied in order to drain it. For a tube with constant radius, the pressure head $h(\mathrm{~m})$ can be related to the radius of the pore $R_{h}$ by (Bear, 1998):

$$
h=\frac{2 T_{s} \cos (\beta)}{\rho g R_{h}},
$$

where $T_{s}\left(\mathrm{~N} \mathrm{~m}^{-1}\right)$ is the surface tension of the water, $\beta$ the contact angle, $\rho\left(\mathrm{kg} \mathrm{m}^{-3}\right)$ the water density and $g\left(\mathrm{~m} \mathrm{~s}^{-2}\right)$ the gravity acceleration. For a drainage experiment, it is then reasonable to consider that the pores with radii between $R_{\min }$ and $R_{h} / a$ will remain fully saturated since we assume that a pore is fully desaturated if its pore throat radius $a R$ is greater than the radius $R_{h}$ (Eq. (7)). Therefore, the main drying effective saturation curve $S_{e}^{d}$ can be expressed as (Soldi et al., 2017):

$$
S_{e}^{d}(h)= \begin{cases}1 & \text { if } \quad h \leq \frac{h_{\min }}{a} \\ \frac{(h a)^{D-2}-h_{\max }^{D-2}}{h_{\min }^{D-2}-h_{\max }^{D-2}} & \text { if } \quad \frac{h_{\min }}{a}<h<\frac{h_{\max }}{a}, \\ 0 & \text { if } \quad h \geq \frac{h_{\max }}{a}\end{cases}
$$

where

$$
h_{\min }=\frac{2 T_{s} \cos (\beta)}{\rho g R_{\max }} \quad h_{\max }=\frac{2 T_{s} \cos (\beta)}{\rho g R_{\min }},
$$

$h_{\min }$ and $h_{\max }$ are the minimum and maximum pressure heads defined by $R_{\max }$ and $R_{\min }$, respectively.

For an imbibition experiment, we consider that the REV is fully desaturated and it is flooded with a pressure head $h$. Then, the pores whose radii are between $R_{\text {min }}$ and $R_{h}$ will 
be fully saturated and the main wetting effective saturation curve $S_{e}^{w}$ can be computed as (Soldi et al., 2017):

$$
S_{e}^{w}(h)= \begin{cases}1 & \text { if } h \leq h_{\min } \\ \frac{h^{D-2}-h_{\max }^{D-2}}{h_{\min }^{D-2}-h_{\max }^{D-2}} & \text { if } \quad h_{\min }<h<h_{\max } . \\ 0 & \text { if } h \geq h_{\max }\end{cases}
$$

Recently, this saturation model has been effectively used to estimate the effect of hysteretic saturation fields on seismic signatures which are generally observed in laboratory during drainage and imbibition experiments (Solazzi et al., 2019).

Similarly, considering the same hypotheses and the Buckingham-Darcy law for unsaturated water flow, Soldi et al. (2017) obtained the main drying relative permeability curve $k_{r e l}^{d}$ as a function of pressure head for a drainage experiment which is given by:

$$
k_{r e l}^{d}(h)= \begin{cases}1 & \text { if } \quad h \leq \frac{h_{\min }}{a} \\ \frac{(h a)^{D-4}-h_{\max }^{D-4}}{h_{\min }^{D-4}-h_{\max }^{D-4}} & \text { if } \quad \frac{h_{\min }}{a}<h<\frac{h_{\max }}{a}, \\ 0 & \text { if } \quad h \geq \frac{h_{\max }}{a}\end{cases}
$$

while for an imbibition experiment, the main wetting relative permeability curve $k_{r e l}^{w}$ can be expressed as:

$$
k_{\text {rel }}^{w}(h)= \begin{cases}1 & \text { if } \quad h \leq h_{\min } \\ \frac{h^{D-4}-h_{\max }^{D-4}}{h_{\min }^{D-4}-h_{\max }^{D-4}} & \text { if } \quad h_{\min }<h<h_{\max } . \\ 0 & \text { if } h \geq h_{\max }\end{cases}
$$

Note that Eqs. (8), (10), (11) and (12) can be used to compute the main drying and wetting curves of the hysteretic cycle observed in the effective saturation and relative permeability. Scanning curves can be scaled from these main curves using different approaches for any intermediate state (e.g., Parker and Lenhard, 1987; Beliaev and Hassanizadeh, 2001). It is important to remark that relative permeability can be expressed as a function of effective saturation which yields in a non-hystertic function (see Ec. (28) from Soldi et al., 2017). This means that the relationship between these two variables $k_{r e l}\left(S_{e}\right)$ is unique for the drying and the wetting, and this result is consistent with observed experimental data (e.g. Topp and Miller, 1966; Van Genuchten, 1980; Mualem, 1986). 


\section{$2.2 \quad$ Electrokinetic properties}

In this section we derive expressions to estimate the electrokinetic phenomenon which results from a coupling between hydraulic and electrokinetic properties at pore scale. We consider that the capillary tubes are saturated by a binary symmetric 1:1 electrolyte (e.g., $\mathrm{NaCl}$ ). Under the hypothesis of a thin double layer (the thickness of the electrical double layer is small compared to the pore radius), the effective excess charge density carried by the water flow in a single tube of constant radius $R$ is given by (Guarracino and Jougnot, 2018):

$$
\hat{Q}_{v}^{R}=\frac{8 N_{A} e_{0} C_{w}^{0}}{\left(R / l_{D}\right)^{2}}\left[-\frac{2 e_{0} \zeta}{k_{B} T}-\left(\frac{e_{0} \zeta}{3 k_{B} T}\right)^{3}\right],
$$

where $N_{A}\left(\mathrm{~mol}^{-1}\right)$ is Avogadro's number, $e_{0}(\mathrm{C})$ the elementary charge, $C_{w}^{0}\left(\mathrm{~mol} \mathrm{~L}^{-1}\right)$ the ionic concentration far from the mineral's surface, $\zeta(\mathrm{V})$ the zeta potential, $k_{B}\left(\mathrm{~J} \mathrm{~K}^{-1}\right)$ the Boltzmann constant, $T(\mathrm{~K})$ the temperature and $l_{D}(\mathrm{~m})$ the Debye length which is defined by:

$$
l_{D}=\sqrt{\frac{\varepsilon k_{B} T}{2 N_{A} C_{w}^{0} e_{0}^{2}}},
$$

being $\varepsilon\left(\mathrm{F} \mathrm{m}^{-1}\right)$ the pore water dielectric permittivity. Note that Eq. (13) is considered valid when the pore radius is greater than $5 l_{D}$ (see Guarracino and Jougnot, 2018; Jougnot et al., 2019).

By assuming the conservation of the electrical charges in the pore volume, the effective excess charge density carried by the water flux in a capillary tube with pore throats $\hat{Q}_{v}^{p}$ $\left(\mathrm{C} \mathrm{m}^{-3}\right)$ can be expressed as:

$$
\hat{Q}_{v}^{p}(R)=\frac{1}{V_{p}} \int_{0}^{l} \hat{Q}_{v}^{r} \pi r^{2}(x) d x=\frac{M}{V_{p}}\left[\int_{0}^{\lambda c} \hat{Q}_{v}^{a R} \pi(a R)^{2} d x+\int_{\lambda c}^{\lambda} \hat{Q}_{v}^{R} \pi R^{2} d x\right]=\hat{Q}_{v}^{R} \frac{1}{f_{v}},
$$

where $V_{p}=\pi R^{2} l f_{v}$ is the volume of a single pore. Note that $\hat{Q}_{v}^{p}$ depends inversely on the factor $f_{v}$ which is a function of the pore geometry parameters, $a$ and $c$ (Eq. (6)). As mentioned in Section 2.1, $f_{v}$ can vary between 0 and 1, and the inverse of this factor is then greater or equal to 1 . Figure 2 shows the effect of the radial factor $a$ on $1 / f_{v}$ for different constant values of the length factor $c(0.1,0.3$ and 0.5$)$. Despite of the fact that $c$ can vary between 0 and 1 , we considered values in the range of 0 to 0.5 for representing realistic pore geometries. It is interesting to observe that as the factor $a$ decreases, the radius of the pore is reduced significantly producing a larger effect on the $\hat{Q}_{v}^{p}$ values. Therefore, the pore throat plays a key role in the estimates of the effective excess charge density.

In order to derive the effective excess charge density $\hat{Q}_{v}^{R E V}$ carried by the water flow in the REV, we consider conditions of saturation similar to those used to compute the hydraulic properties (Section 2.1). For a drainage test, we assume that a pressure head $h$ is applied to drain a fully saturated REV. Then, only the pores that remain fully saturated $\left(R_{\min } \leq R \leq R_{h} / a\right)$ contribute to the volumetric water flow, and hence to the effective 
with Eqs. (5) and (8) yields:

$$
\hat{Q}_{v}^{R E V, d}=N_{A} e_{0} C_{w}^{0}\left[-\frac{2 e_{0} \zeta}{k_{B} T}-\left(\frac{e_{0} \zeta}{3 k_{B} T}\right)^{3}\right] \frac{l_{D}^{2}}{\tau^{2}} \frac{f_{k}}{f_{v}^{2}} \frac{\phi}{k} \frac{S_{e}^{d}}{k_{r e l}^{d}} .
$$

Similarly, for an imbibition test, we consider that the REV is saturated with a pressure head $h$. The pores with radius smaller than $R_{h}$ will be fully saturated and thus contribute to the water flow. Then, the effective excess charge density $\hat{Q}_{v}^{R E V, w}$ can be expressed as:

$$
\hat{Q}_{v}^{R E V, w}=N_{A} e_{0} C_{w}^{0}\left[-\frac{2 e_{0} \zeta}{k_{B} T}-\left(\frac{e_{0} \zeta}{3 k_{B} T}\right)^{3}\right] \frac{l_{D}^{2}}{\tau^{2}} \frac{f_{k}}{f_{v}^{2}} \frac{\phi}{k} \frac{S_{e}^{w}}{k_{r e l}^{w}} .
$$

Note that in the case of non-constrictive tubes, $a=1$ (or $c=0$ ) which yields to $f_{v}=f_{k}=$ 1, Eqs. (19) and (20) have the same analytical expression which is the equation obtained by Soldi et al. (2019) for the effective excess charge density in tortuous straight tubes.

Equations (19) and (20) can be expressed as:

$$
\hat{Q}_{v}^{R E V, i}=\hat{Q}_{v}^{R E V, s a t} \hat{Q}_{v}^{R E V, r e l, i}
$$

4 where the effective excess charge density for saturated conditions $\hat{Q}_{v}^{R E V \text {,sat }}\left(\mathrm{C} \mathrm{m}^{-3}\right)$ is given by:

$$
\hat{Q}_{v}^{R E V, s a t}=N_{A} e_{0} C_{w}^{0}\left[-\frac{2 e_{0} \zeta}{k_{B} T}-\left(\frac{e_{0} \zeta}{3 k_{B} T}\right)^{3}\right] \frac{l_{D}^{2}}{\tau^{2}} \frac{f_{k}}{f_{v}^{2}} \frac{\phi}{k}
$$

and the relative effective excess charge density $\hat{Q}_{v}^{R E V, r e l}$ (dimensionless) by:

$$
\hat{Q}_{v}^{R E V, r e l, i}=\frac{S_{e}^{i}}{k_{r e l}^{i}}
$$




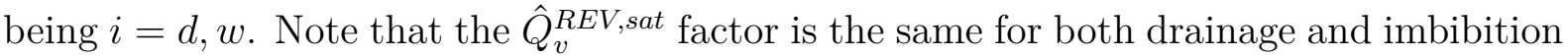
experiments and depends on petrophysical and electro-chemical properties. However, the $\hat{Q}_{v}^{R E V, r e l, i}$ factor only depends on the hydraulic properties of the media which differ between drainage and imbibition tests. Then, the hysteresis phenomenon in the effective excess charge density is associated with the relative factor.

By inspection of Eq. (23), it can be noticed that when $S_{e}$ approaches zero, both terms of the quotient tend to zero for drainage (when $h \rightarrow h_{\max } / a$ ) and imbibition (when $h \rightarrow h_{\max }$ ). From Eqs. (8), (11), (10) and (12), we obtain the same asymptotic value for drainage and imbibition:

$$
\lim _{S_{e} \rightarrow 0} \hat{Q}_{v}^{R E V, r e l, i}=\lim _{h \rightarrow \frac{h_{\max }}{a}} \frac{S_{e}^{d}(h)}{k_{r e l}^{d}(h)}=\lim _{h \rightarrow h_{\max }} \frac{S_{e}^{w}(h)}{k_{r e l}^{w}(h)}=\frac{h_{\min }^{D-4}-h_{\max }^{D-4}}{h_{\min }^{D-2}-h_{\max }^{D-2}} .
$$

This limit case represents the excess charge of the pores with smallest radius dragged by the residual water saturation.

The main drying relative effective excess charge density curve $\hat{Q}_{v}^{R E V, r e l, d}$ as a function of the pressure head $h$ can be obtained by substituting Eqs. (8) and (11) into Eq. (23):

$$
\hat{Q}_{v}^{R E V, r e l, d}(h)= \begin{cases}1 & \text { if } h \leq \frac{h_{\min }}{a} \\ \frac{(h a)^{D-2}-h_{\max }^{D-2}}{(h a)^{D-4}-h_{\max }^{D-4}} \cdot \frac{h_{\min }^{D-4}-h_{\max }^{D-4}}{h_{\min }^{D-2}-h_{\max }^{D-2}} & \text { if } \quad \frac{h_{\min }}{a}<h<\frac{h_{\max }}{a} . \\ \frac{h_{\min }^{D-4}-h_{\max }^{D-4}}{h_{\min }^{D-2}-h_{\max }^{D-2}} & \text { if } \quad h \geq \frac{h_{\max }}{a}\end{cases}
$$

Similarly, the main wetting relative effective excess charge density curve $\hat{Q}_{v}^{R E V, r e l, w}$ is obtained by replacing Eqs. (10) and (12) into Eq. (23):

$$
\hat{Q}_{v}^{R E V, r e l, w}(h)= \begin{cases}1 & \text { if } h \leq h_{\min } \\ \frac{h^{D-2}-h_{\max }^{D-2}}{h^{D-4}-h_{\max }^{D-4}} \cdot \frac{h_{\min }^{D-4}-h_{\max }^{D-4}}{h_{\min }^{D-2}-h_{\max }^{D-2}} & \text { if } \quad h_{\min }<h<h_{\max } . \\ \frac{h_{\min }^{D-4}-h_{\max }^{D-4}}{h_{\min }^{D-2}-h_{\max }^{D-2}} & \text { if } h \geq h_{\max }\end{cases}
$$

Note that the relative effective excess charge density expressions for both drying and wetting have analytical closed form expressions which depend on independent parameters ( $a, D, h_{\min }$ and $h_{\max }$ ) with geometrical and physical meaning.

\section{Sensitivity analysis of the model}

In order to study the role of the model parameters in the estimates of the relative effective excess charge density, we perform a parametric analysis of Eqs. (25) and (26). We test the influence of the fractal dimension $D$, the radial factor $a$ that controls the pore throats 


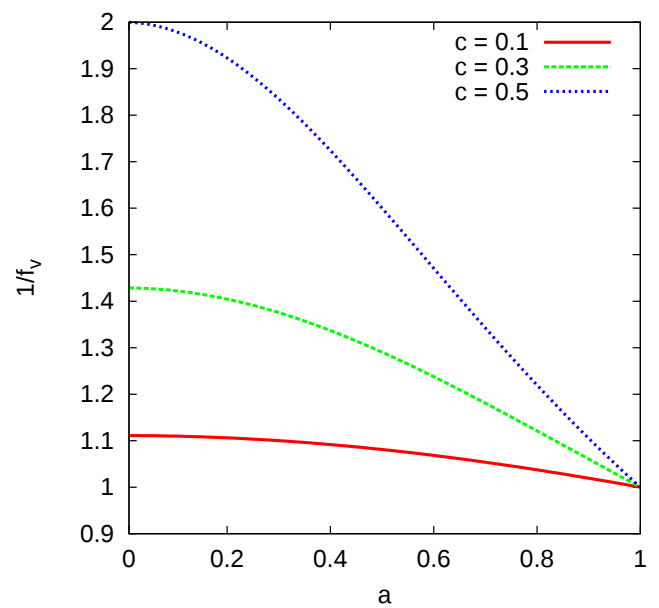

Figure 2: Dimensionless factor $1 / f_{v}$ as a function of the radial factor $a$ for different constant values of parameter $c$.

and the minimum pore size $R_{\min }$ as these parameters produce the greatest impact in the size distribution and geometry of the porous media. The following reference values of these parameters are considered: $D=1.5, a=0.5$ and $R_{\min }=1.5 \times 10^{-4} \mathrm{~mm}$.

Figure 3 summarizes this analysis and shows the curves of the hydraulic properties $S_{e}$ and $k_{r e l}$ (Eqs. (8), (10), (11) and (12)) besides the relative effective excess charge density curves (Eqs. (25) and (26)). Figures 3a-c show the effect of the fractal dimension for fixed values of the other parameters. It can be observed that parameter $D$ produces significant differences between the hysteretic loops of the effective saturation curves, while it slightly affects the loops of the relative permeability. For high pressure head values, no significant variations are shown among the $S_{e}$ and $k_{r e l}$ curves, nevertheless, the asymptotic values of the relative effective excess charge density vary with the different values of parameter $D$ (see Fig. 3c). In fact, note that the maximum value of $\hat{Q}_{v}^{R E V, r e l}$ increases when $D$ decreases, however, this variation remains within one order of magnitude. Figures 3d-f show the effect of the radial factor $a$. The influence of this parameter is significant in the main drying curves of effective saturation and relative permeability for the entire range of pressure head values. However, no variations are observed in the main wetting curves of the hydraulic properties since they are independent of $a$, and hence these curves are overlapping each other for the different values of $a$. As a result, this parameter strongly affects only the main drying $\hat{Q}_{v}^{R E V \text {,rel }}$ curve for all the pressure head values. Indeed, the

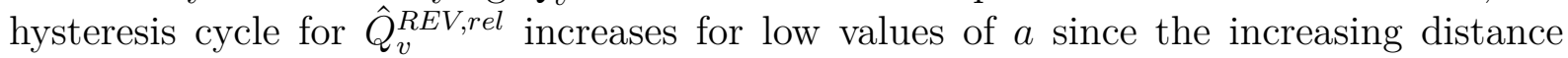
between the curves of the drainage and imbibition experiments (see Fig. 3f). As a tends toward 1, the two main $\hat{Q}_{v}^{R E V \text {,rel }}$ curves tend to reduce their distance, as it can be expected since this limit case represents a tube of constant radius and thus no hysteretic phenomenon will be observed. Figures $3 \mathrm{~g}$-i show the effect of $R_{\min }$, this parameter is inversely proportional to $h_{\max }$ (Eq. (7)). The effect of $R_{\min }$ is significant in the $S_{e}$ hysteretic loops for increasing values of pressure head, while it is not significant in the $k_{r e l}$ curves. Hence, the $\hat{Q}_{v}^{R E V, r e l}$ curves show the strongest differences for high values of 

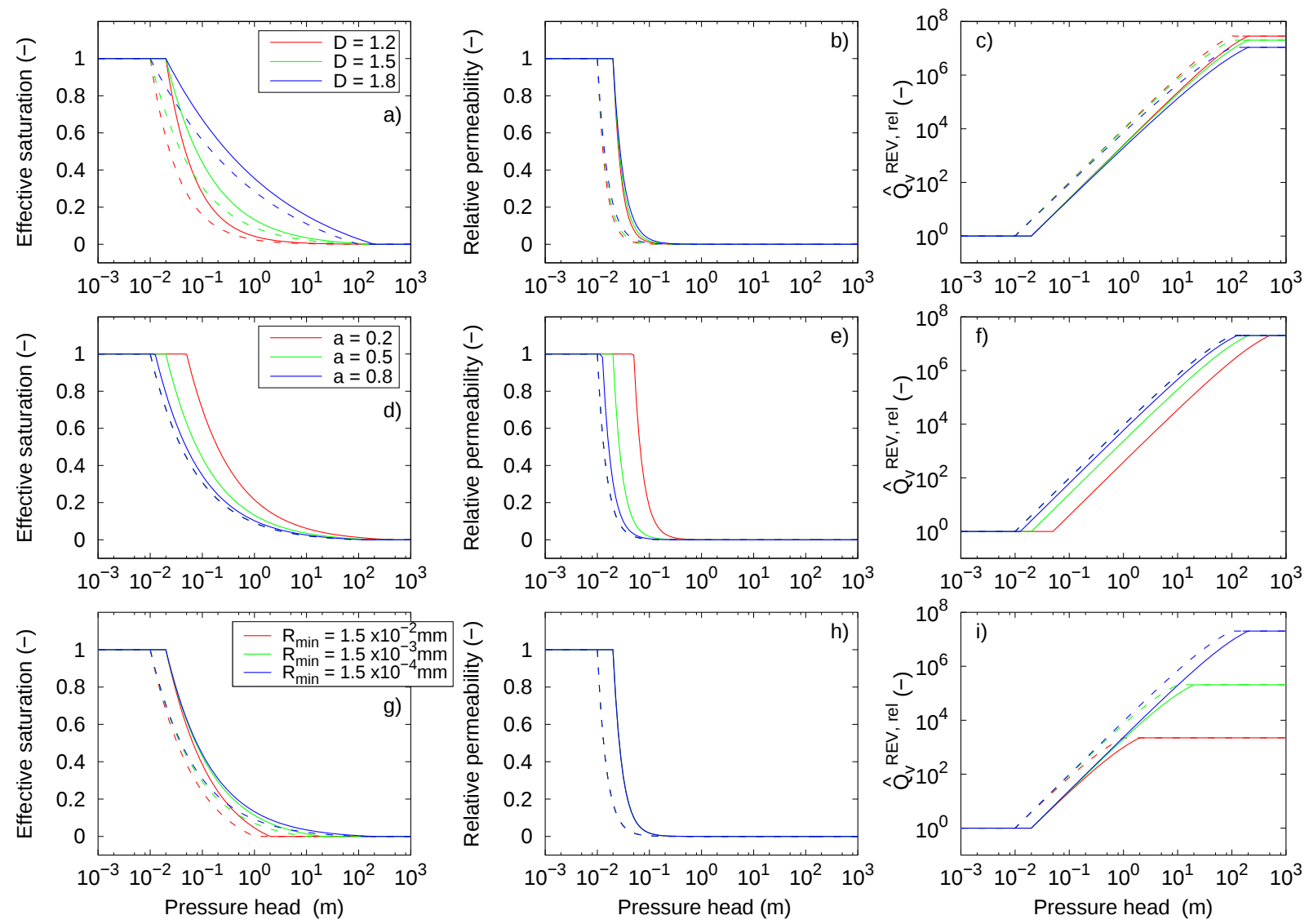

Figure 3: Parametric analysis of the relative effective excess charge density $\hat{Q}_{v}^{R E V, r e l}$ for drainage (solid lines) and imbibition (dashed lines): c) sensitivity to the fractal dimension $D, \mathrm{f})$ sensitivity to the radial factor $a$, and i) sensitivity to the minimum radius $R_{\text {min }}$ (which corresponds to values of $h_{\max }$, see Eq. (7)). Note that fixed values of the remaining parameters were considered in each case. The corresponding curves of effective saturation (Fig.3(a), 3(d) and 3(g)) and relative permeability (Fig.3(b), 3(e) and 3(h)) are also shown.

pressure head. It can also be observed that the maximum value of $\hat{Q}_{v}^{R E V, r e l}$ increases when $R_{\min }$ decreases (see Fig. 3i) since, at residual water saturation, the pores with smaller radius are the ones that remain with water and a significant amount of excess charge is dragged. In addition, note that this parameter can change $\hat{Q}_{v}^{R E V, r e l}$ in 3 orders of magnitude while the distance between the main drying and wetting curves of the loops remains approximately constant.

Finally, from this parametric analysis, we can conclude that parameters $a$ and $R_{\min }$ produce the most significant changes in the estimates of $\hat{Q}_{v}^{R E V, r e l}$. Furthermore, while the estimates of the main drying $\hat{Q}_{v}^{R E V, r e l}$ curve are highly sensitive to parameter $a$ which produces strong differences between this curve and the wetting $\hat{Q}_{v}^{R E V, r e l}$ curve, parameter $R_{\text {min }}$ can modify $\hat{Q}_{v}^{R E V, r e l}$ values over several orders of magnitude. 


\section{Relative coupling coefficient}

The effective excess charge is an efficient parameter to study the electrokinetic coupling under partially saturated conditions. This parameter is the basis of an approach that has been increasingly employed in the last decades, nevertheless, the Helmholtz-Smoluchowski approach is the most used in the literature which is based on the coupling coefficient $C_{E K}$. This coefficient relates an electrical potential difference and a hydraulic pressure head difference generated by the water flow. The relationship between the relative coupling coefficient $C_{E K}^{r e l}$ and the relative effective excess charge density $\hat{Q}_{v}^{R E V, r e l}$ can be obtained from Eq. (1) (e.g. Linde et al., 2007; Revil et al., 2007):

$$
C_{E K}^{r e l, i}=\frac{\hat{Q}_{v}^{R E V, r e l, i} k_{r e l}^{i}}{\sigma^{r e l}}
$$

where Eqs. (11) and (19) are used to calculate this parameter for the drainage case, and Eqs. (12) and (20) for the imbibition case, whereas the relative electrical conductivity is estimated using Archie's second law (Archie et al., 1942), $\sigma^{r e l}=S_{w}^{n}$ being $n$ the water saturation exponent. In this sensitivity analysis, we consider a simple non-hysteretic model for the electrical conductivity to better focus on the hysteresis in the effective excess charge density function. We then considered two different soil textures to study the estimates and behaviour of the coupling parameter within the framework of the approach based on the effective excess charge and the Helmholtz-Smoluchowski approach. The soil textures are a sand and a silt which were used by Soldi et al. (2017) to estimate the hysteretic saturation from the experimental data from Pham et al. (2003). Table 1 lists the parameters used to estimate the hydraulic and electrical properties of the two textures. The hydraulic parameters were taken from Soldi et al. (2017) for both textures, while the electrical parameter was taken from Lesmes and Friedman (2005) and Doussan and Ruy (2009) for the sand and silt respectively.

Fig. 4 shows the relative effective excess charge density $\hat{Q}_{v}^{R E V \text {,rel }}$ and the relative coupling coefficient $C_{E K}^{r e l}$ as functions of both the pressure head $h$ and effective water saturation $S_{e}$ for the two different soil textures. It is interesting to note that the hysteretic effect on both $\hat{Q}_{v}^{R E V \text {,rel }}$ and $C_{E K}^{r e l}$ can be observed when these parameters vary with pressure head values (see Figs. 4a and 4c). However, when they are represented as a function of $S_{e}$, the resulting curve is non-hysteretic as shown in Figs. 4b and 4d. For a fixed value of pressure head, the estimates of $\hat{Q}_{v}^{R E V \text {,rel }}$ vary significantly between the two soil textures (being the greater values for the sand) while the differences in the estimates of $C_{E K}^{r e l}$ are smaller between the two textures. It can also be observed that whereas the $\hat{Q}_{v}^{R E V \text {,rel }}$ values vary over several orders of magnitude (about 2 and 6 orders for the silt and the sand, respectively), the $C_{E K}^{r e l}$ values remain within the range $0 \sim 1.1$ for the two soil textures. For all the effective saturation range, the $C_{E K}^{r e l}$ curve for the silt remains below the corresponding curve for the sand. Nevertheless, the estimates of $\hat{Q}_{v}^{R E V \text {,rel for }}$ the silt are smaller than the estimates for the sand only for low saturation values.

In a recent study, Zhang et al. (2017) proposed a model to determine the saturation dependence of the relative coupling coefficient and observed from that relationship that 

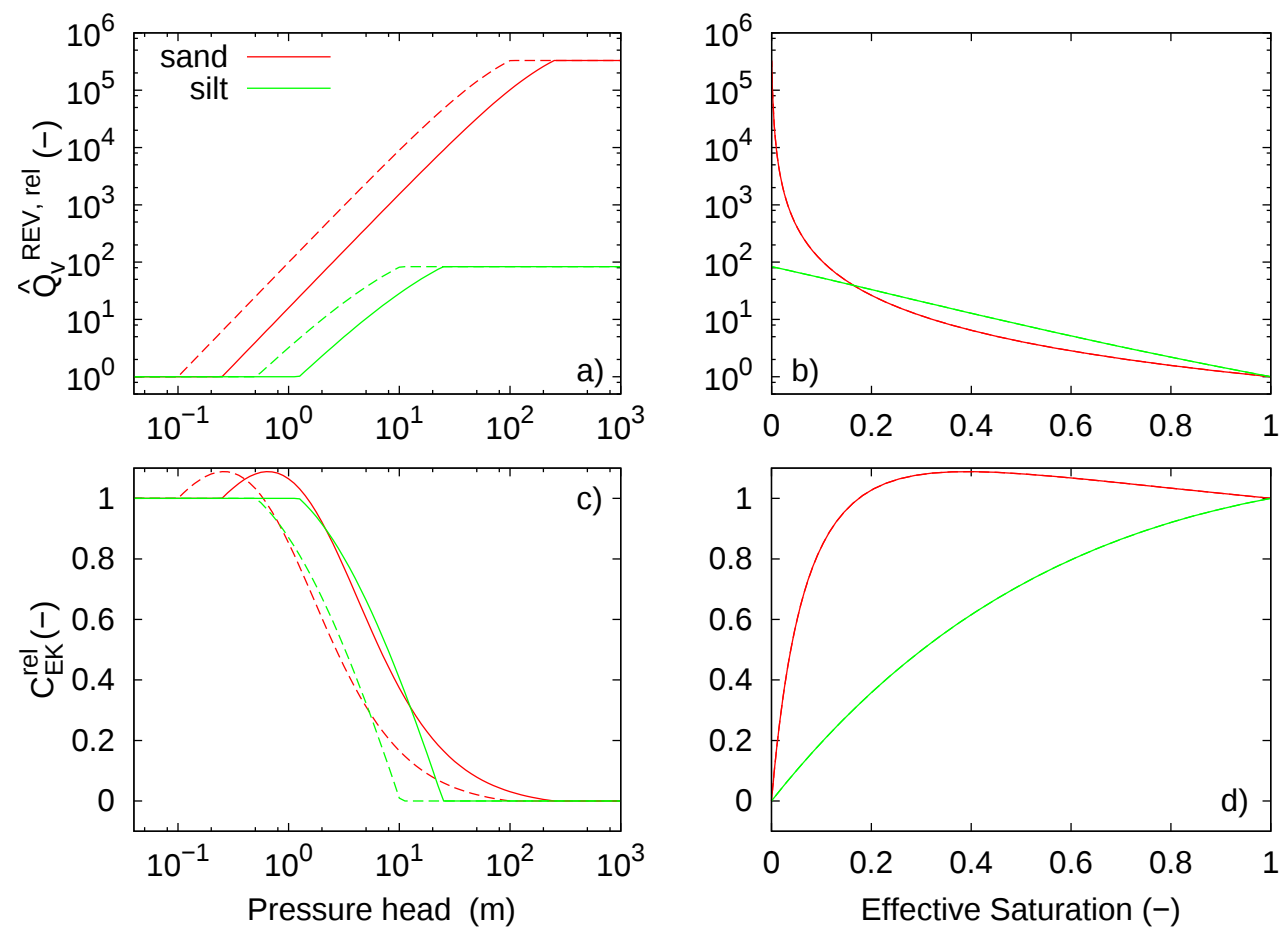

Figure 4: (a,b) Relative effective excess charge density and (c,d) relative coupling coefficient as functions of pressure head and effective saturation for two soil textures: a sand and a silt. The solid and dashed lines in Figs.4(a) and 4(c) correspond to the drainage and imbibition cases, respectively.

$C_{E K}^{r e l}$ exhibits hysteresis. Such behaviour can not be explained by the model developed in this study as the resulting $C_{E K^{-}}^{r e l} S_{e}$ curve is non-hysteretic. The hysteresis observed by Zhang et al. (2017) could be attributed to the numerical approximations used to calculate water saturation or other phenomena such as changes in wettability or entrapped air. From a theoretical point of view, no hysteresis phenomenon is present in $C_{E K}^{r e l}$ when expressed as a function of effective saturation. Note that the behaviour of the proposed $C_{E K^{-}}^{r e l} S_{e}$ curves, shown in Fig. 4d, is consistent with previous works considering the coupling coefficient (e.g., Bordes et al., 2015) and theoretical models assuming the porous media as bundles of capillary tubes (see Figs. 6c and 6d from Jougnot et al., 2012). The model of Jackson (2010) predicted a decrease in the estimates of $C_{E K}^{r e l}$ when decreasing $S_{e}$. In addition, the model derived by Jougnot et al. (2012) also predicted that the $C_{E K}^{r e l}$ values decrease when $S_{e}$ decreases. Moreover, they observed strong differences on the $\hat{Q}_{v}^{R E V, r e l}$ and $C_{E K}^{r e l}$ estimates as functions of $S_{e}$ for different soil textures (see their Fig. 6). They also showed that $C_{E K}^{r e l}$ can reach values greater than 1 for low effective saturation values when considering a sand texture, but that it remains smaller than 1 for a silt. As shown in Fig. 4d, this behaviour of $C_{E K}^{r e l}$ for those two textures is also predicted by the proposed model. 
Table 1: Values of the parameters used to estimate the relative effective excess charge density and the relative coupling coefficient for a sand and a silt.

\begin{tabular}{lccccc}
\hline Soil type & \multicolumn{4}{c}{ Proposed model parameters* } & Electrical parameter \\
& $D(-)$ & $a(-)$ & $h_{\min }(\mathrm{m})$ & $h_{\max }(\mathrm{m})$ & $n(-)$ \\
\hline Sand & 1.02 & 0.40 & 0.112 & 100.00 & 1.30 \\
Silt & 1.76 & 0.41 & 0.510 & 10.20 & 5.96 \\
\hline
\end{tabular}

*Values taken from Soldi et al. (2017).

+The sand value was taken from Lesmes and Friedman (2005), while the silt value from Doussan and Ruy (2009).

\section{Comparison with experimental data}

Data sets of coupling coefficient-pressure head for drainage and imbibition experiments are lacking thus far in the literature. Allègre et al. (2014) studied the self-potential (SP) response to a periodic succession of drainage and imbibition cycles in a column filled with clean Fontainbleau sand. They measured values of pressure head $h$ at two different points ( $h_{1}$ and $h_{2}$ ) of the column and the SP differences $\Delta V$ between them. In order to test the proposed model, we estimated relative coupling coefficient values $C_{E K}^{r e l}$ from the recorded data (see Fig. 4 from Allègre et al., 2014) as a function of the mean pressure head value between the two points as follows:

$$
C_{E K}^{r e l}\left(h_{1}+\frac{\Delta h}{2}\right)=\frac{1}{C_{E K}^{s a t}} \frac{\Delta V}{\Delta h},
$$

where $\Delta h=h_{2}-h_{1}$ corresponds to the pressure head differences between the points. For $C_{E K}^{s a t}$, we considered the value measured by Allègre et al. (2014) for the sand column under total saturation conditions.

Figure 5 shows the $C_{E K}^{r e l}$ data obtained from Allègre et al. (2014) using Eq. (28) and the relative coupling coefficient model for the sand estimated previously in Section 4. The data show high scattering, nevertheless, it can be observed that the behaviour shown by the $C_{E K}^{r e l}$ data values is different for the drainage and for the imbibition experiments. Even so, it is not possible to establish a clear pattern of the data in either of the cases. Note also that the $C_{E K}^{r e l}$ values of the experimental data reach values greater than 1 as predicted by the proposed model.

\section{Discussion and conclusion}

A physically based theoretical model to describe hysteresis phenomenon in the estimates of the effective excess charge density for partially saturated conditions has been presented. 


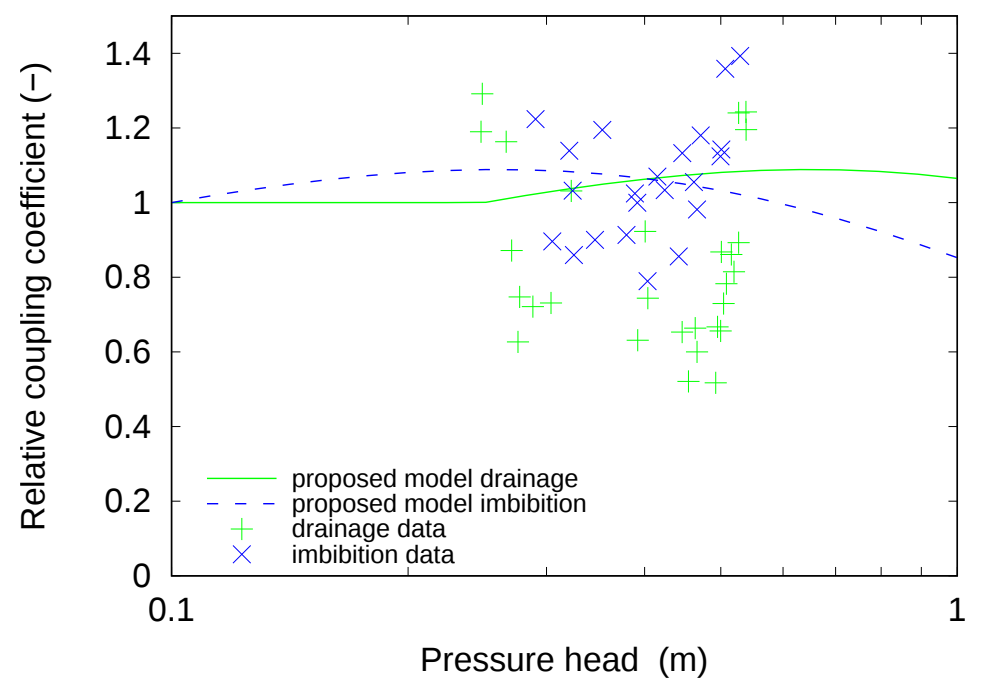

Figure 5: Relative coupling coefficient as a function of pressure head for experimental data from a sand sample from Allègre et al. (2014).

The proposed model is based on the assumption that the porous medium can be represented by a bundle of tortuous capillary tubes with periodic pore throats. The derivation of the model involved upscaling procedures at pore and REV scales of the hydraulic and electrokinetic properties of the porous medium. Considering a fractal distribution of pore sizes and a binary symmetric 1:1 electrolyte, analytical closed-form expressions have been obtained for the effective excess charge density $\hat{Q}_{v}^{R E V}$ for the drainage and imbibition experiments.

The hysteretic behaviour of the effective excess charge density is explicitly observed in the relative factor $\hat{Q}_{v}^{R E V, r e l}$ when expressed as a function of pressure head since it depends on the flow history of the medium. This phenomenon is easily introduced in the model by the presence of the pore throats as it strongly controls the flow properties of the medium. The radial factor $a$ plays a key role to represent the hysteresis in the proposed model as it controls the size of the pore throats. In addition, if $a=1$ (pores with constant radii), the expression of the proposed model becomes the expression proposed by Soldi et al. (2019) for non-constrictive tortuous capillaries.

The saturated effective excess charge density factor $\hat{Q}_{v}^{R E V \text {,sat }}$ depends on the petrophysical properties of the medium and the chemical parameters of the pore water while also being affected by the presence of the pore throats through the factor $f_{k} / f_{v}^{2}$. This factor depends on the radial $a$ and length $c$ factors of the pore throat. In the limit case

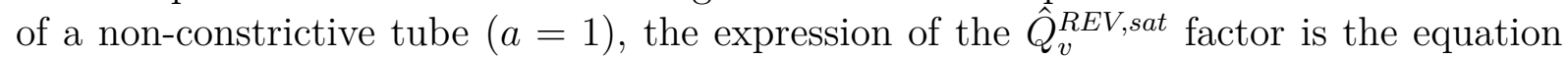
obtained by Soldi et al. (2019) for saturated conditions.

The influence of the model parameters $\left(D, a\right.$ and $\left.R_{\text {min }}\right)$ on the estimates of the relative effective excess charge density has been tested by a sensitivity analysis. The results show that variations of the fractal dimension $D$ slightly affect the $\hat{Q}_{v}^{R E V \text {,rel }}$ estimates. Nevertheless, the effects of the radial factor $a$ and the minimum pore radius $R_{\text {min }}$ produce 
the most significant variations in the $\hat{Q}_{v}^{R E V, r e l}$ values. The factor $a$ controls the shape of the hysteretic loop (the distance between the drainage and imbibition curves), and in the limit case of $a=1$, the hysteresis disappears from the $\hat{Q}_{v}^{R E V \text {,rel }}$ curves as it will be expected. Nonetheless, the variations of $R_{\min }$ can affect the $\hat{Q}_{v}^{R E V \text {,rel }}$ estimates over several orders of magnitude.

The comparison of the relative effective excess charge density and the relative coupling coefficient estimates for two different soil textures shows that both parameters exhibit the hysteresis phenomenon when expressed as functions of the pressure head. However, a non-hysteretic behaviour is observed when they are described as functions of the effective saturation. The comparison of the two soil textures also shows significant differences in

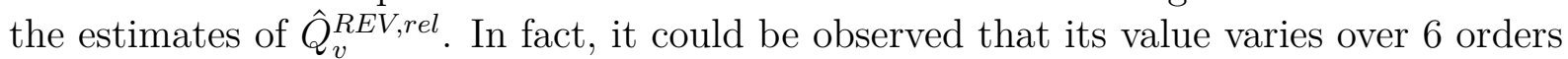
of magnitude for the sand while over 2 orders for the silt. Nevertheless, the $C_{E K}^{r e l}$ values vary in a small range (0 1.1 approximately) for both textures.

To the best of our knowledge, the data shown in Figure 5 are the only hysteretic data available to validate coupling coefficient curves as function of pressure head values. From a qualitative comparison, the $C_{E K}^{r e l}$ values estimated from the proposed model are consistent with the experimental data values. Even though, further drainage-imbibition tests are needed, the proposed model provides a simple and physically meaningful way to include hysteresis effects on the electrokinetic potential.

Based on the framework of the effective excess charge, the present study represents a step forward to understand the electrokinetic coupling under partially saturated conditions since the model includes hysteresis phenomenon in SP signals. As far as reported in literature, this is the first analytical model that accounts this phenomenon in the streaming potential. Therefore, this simple model can be a valuable starting point to the use of the SP method in hydrogeophysics studies to non-intrusively monitor unsaturated groundwater fluxes (e.g., Doussan et al., 2002; Suski et al., 2006; Jougnot et al., 2015; Voytek et al., 2019; Hu et al., 2020) and help to improve the understanding of processes occurring in the vadose zone, such as contaminant plumes (e.g. Naudet et al., 2003; Minsley et al., 2007), hydro-fracturing (e.g. Darnet et al., 2006; Haas et al., 2013) or related to reservoir engineering (Saunders et al., 2006).

\section{Acknowledgments}

This research is partially supported by Universidad Nacional de La Plata, Consejo Nacional de Investigaciones Científicas y Técnicas (Argentina), Sorbonne Université and Centre National de la Recherche Scientifique (France). The authors strongly thank the Editor and an anonymous reviewer for their constructive comments. D.J. strongly thanks the financial support of the CNRS INSU EC2CO program for funding the STARTREK (Système péTrophysique de cAractéRisation du Transport Réactif en miliEu Karstique) project. authoryear 


\section{References}

Allègre, V., Maineult, A., Lehmann, F., Lopes, F., Zamora, M., 2014. Self-potential response to drainage-imbibition cycles. Geophysical Journal International 197, 14101424.

Archie, G.E., et al., 1942. The electrical resistivity log as an aid in determining some reservoir characteristics. Transactions of the AIME 146, 54-62.

Bear, J., 1998. Dynamics of fluids in porous media. Dover Publications, Inc., Mineola, N.Y.

Beliaev, A.Y., Hassanizadeh, S., 2001. A theoretical model of hysteresis and dynamic effects in the capillary relation for two-phase flow in porous media. Transport in Porous media $43,487-510$.

Bordes, C., Sénéchal, P., Barrì̀re, J., Brito, D., Normandin, E., Jougnot, D., 2015. Impact of water saturation on seismoelectric transfer functions: a laboratory study of coseismic phenomenon. Geophysical Journal International 200, 1317-1335.

Buckingham, E., 1907. Studies on the movement of soil moisture. US Dept. Agic. Bur. Soils Bull. 38.

Büsing, H., Vogt, C., Ebigbo, A., Klitzsch, N., 2017. Numerical study on co2 leakage detection using electrical streaming potential data. Water Resources Research 53, 455469.

Darcy, H., 1856. Exposition et application des principes à suivre et des formules à employer dans les questions de distribution d'eau. Les fontaines publiques de la ville de Dijon, Eds. Victor Dalmont, Paris 1856.

Darnet, M., Marquis, G., Sailhac, P., 2006. Hydraulic stimulation of geothermal reservoirs: fluid flow, electric potential and microseismicity relationships. Geophysical Journal International 166, 438-444.

Doussan, C., Jouniaux, L., Thony, J.L., 2002. Variations of self-potential and unsaturated water flow with time in sandy loam and clay loam soils. Journal of Hydrology 267, 173185.

Doussan, C., Ruy, S., 2009. Prediction of unsaturated soil hydraulic conductivity with electrical conductivity. Water Resources Research 45, W10408, doi:10.1029/2008WR007309.

Fan, Y., Grant, G., Anderson, S.P., 2019. Water within, moving through, and shaping the earth's surface: Introducing a special issue on water in the critical zone. Hydrological Processes, https://onlinelibrary.wiley.com/doi/abs/10.1002/hyp.13638. 
Glover, P.W., Déry, N., 2010. Streaming potential coupling coefficient of quartz glass bead packs: Dependence on grain diameter, pore size, and pore throat radiusstreaming potential and grain diameter. Geophysics 75, F225-F241.

Guarracino, L., Jougnot, D., 2018. A physically based analytical model to describe effective excess charge for streaming potential generation in water saturated porous media. Journal of Geophysical Research: Solid Earth 123, 52-65.

Guarracino, L., Rötting, T., Carrera, J., 2014. A fractal model to describe the evolution of multiphase flow properties during mineral dissolution. Advances in water resources $67,78-86$.

Guichet, X., Jouniaux, L., Pozzi, J.P., 2003. Streaming potential of a sand column in partial saturation conditions. Journal of Geophysical Research: Solid Earth 108.

Haas, A., Revil, A., 2009. Electrical burst signature of pore-scale displacements. Water Resources Research 45.

Haas, A., Revil, A., Karaoulis, M., Frash, L., Hampton, J., Gutierrez, M., Mooney, M., 2013. Electric potential source localization reveals a borehole leak during hydraulic fracturing. Geophysics 78, D93-113.

Hu, K., Jougnot, D., Huang, Q., Looms, M.C., Linde, N., 2020. Advancing quantitative understanding of self-potential signatures in the critical zone through long-term monitoring. Journal of Hydrology 585, 124771.

Hunter, R., 1981. Zeta potential in colloid science: Principles and applications. New York, USA .

Jackson, M.D., 2008. Characterization of multiphase electrokinetic coupling using a bundle of capillary tubes model. Journal of Geophysical Research: Solid Earth 113, doi:10.1029/2007JB005490.

Jackson, M.D., 2010. Multiphase electrokinetic coupling: Insights into the impact of fluid and charge distribution at the pore scale from a bundle of capillary tubes model. Journal of Geophysical Research: Solid Earth 115, doi:10.1029/2009JB007092.

Jardani, A., Dupont, J.P., Revil, A., 2006. Self-potential signals associated with preferential groundwater flow pathways in sinkholes. Journal of Geophysical Research: Solid Earth 111, doi:10.1029/2005JB004231.

Jerauld, G., Salter, S., 1990. The effect of pore-structure on hysteresis in relative permeability and capillary pressure: pore-level modeling. Transport in porous media 5, $103-151$.

Jougnot, D., Linde, N., 2013. Self-potentials in partially saturated media: the importance of explicit modeling of electrode effects. Vadose Zone Journal 12. 
Jougnot, D., Linde, N., Haarder, E.B., Looms, M.C., 2015. Monitoring of saline tracer movement with vertically distributed self-potential measurements at the hobe agricultural test site, voulund, denmark. Journal of Hydrology 521, 314-327.

Jougnot, D., Linde, N., Revil, A., Doussan, C., 2012. Derivation of soil-specific streaming potential electrical parameters from hydrodynamic characteristics of partially saturated soils. Vadose Zone Journal 11, doi:10.2136/vzj2011.0086.

Jougnot, D., Mendieta, A., Leroy, P., Maineult, A., 2019. Exploring the effect of the pore size distribution on the streaming potential generation in saturated porous media, insight from pore network simulations. Journal of Geophysical Research: Solid Earth 124, 5315-5335, https://doi.org/10.1029/2018JB017240.

Jougnot, D., Roubinet, D., Guarracino, L., Maineult, A., 2020. Modeling streaming potential in porous and fractured media, description and benefits of the effective excess charge density approach, in: Advances in Modeling and Interpretation in Near Surface Geophysics. Springer, pp. 61-96.

Jury, W., Gardner, W.R., Gardner, W.H., 1991. Soil physics, john wiley \& sons. Inc. New York .

Klausner, Y., 1991. Fundamentals of Continuum Mechanics of Soils. Springer, New York.

Kormiltsev, V.V., Ratushnyak, A.N., Shapiro, V.A., 1998. Three-dimensional modeling of electric and magnetic fields induced by the fluid flow movement in porous media. Physics of the earth and planetary interiors 105, 109-118.

Lenhard, R., Parker, J., 1987. A model for hysteretic constitutive relations governing multiphase flow: 2. permeability-saturation relations. Water Resources Research 23, $2197-2206$.

Lesmes, D.P., Friedman, S.P., 2005. Relationships between the electrical and hydrogeological properties of rocks and soils, in: Hydrogeophysics. Springer, New York, pp. $87-128$

Linde, N., 2009. Comment on "characterization of multiphase electrokinetic coupling using a bundle of capillary tubes model" by mathew d. jackson. Journal of Geophysical Research: Solid Earth 114, doi:10.1029/2008JB005845.

Linde, N., Jougnot, D., Revil, A., Matthäi, S., Arora, T., Renard, D., Doussan, C., 2007. Streaming current generation in two-phase flow conditions. Geophysical Research Letters 34, L03306. doi:10.1029/2006GL028878.

Linde, N., Revil, A., 2007. Inverting self-potential data for redox potentials of contaminant plumes. Geophysical Research Letters 34, L14302. https://doi.org/10.1029/2007GL030084. 
Maineult, A., Strobach, E., Renner, J., 2008. Self-potential signals induced by periodic pumping tests. Journal of Geophysical Research: Solid Earth 113, doi:10.1029/2007JB005193.

Malama, B., Kuhlman, K.L., Revil, A., 2009a. Theory of transient streaming potentials associated with axial-symmetric flow in unconfined aquifers. Geophysical Journal International 179, 990-1003.

Malama, B., Revil, A., Kuhlman, K., 2009b. A semi-analytical solution for transient streaming potentials associated with confined aquifer pumping tests. Geophysical Journal International 176, 1007-1016.

Minsley, B.J., Sogade, J., Morgan, F.D., 2007. Three-dimensional self-potential inversion for subsurface dnapl contaminant detection at the savannah river site, south carolina. Water Resources Research 43. doi:10.1029/2005WR003996.

Morgan, F., Williams, E., Madden, T., 1989. Streaming potential properties of westerly granite with applications. Journal of Geophysical Research: Solid Earth 94, 1244912461.

Mualem, Y., 1977. Extension of the similarity hypothesis used for modeling the soil water characteristics. Water Resources Research 13, 773-780.

Mualem, Y., 1986. Hydraulic conductivity of unsaturated soils: prediction and formulas. Methods of Soil Analysis: Part 1-Physical and Mineralogical Methods , 799-823.

Naudet, V., Revil, A., Bottero, J.Y., Bégassat, P., 2003. Relationship between selfpotential (sp) signals and redox conditions in contaminated groundwater. Geophysical research letters 30. doi:10.1029/2003GL018096.

Parker, J., Lenhard, R., 1987. A model for hysteretic constitutive relations governing multiphase flow: 1. saturation-pressure relations. Water Resources Research 23, 21872196.

Petiau, G., 2000. Second generation of lead-lead chloride electrodes for geophysical applications. Pure and applied geophysics 157, 357-382.

Pham, H., Fredlund, D., Barbour, S., 2003. A practical hysteresis model for the soil-water characteristic curve for soils with negligible volume change. Geotechnique 53, 293-298.

Pham, H.Q., Fredlund, D.G., Barbour, S.L., 2005. A study of hysteresis models for soil-water characteristic curves. Canadian Geotechnical Journal 42, 1548-1568.

Revil, A., 2017. Transport of water and ions in partially water-saturated porous media. part 1. constitutive equations. Advances in water resources 103, 119-138, https://doi.org/10.1016/j.advwatres.2016.02.006. 
Revil, A., Gevaudan, C., Lu, N., Maineult, A., 2008. Hysteresis of the self-potential response associated with harmonic pumping tests. Geophysical Research Letters 35, doi:10.1029/2008GL035025.

Revil, A., Linde, N., Cerepi, A., Jougnot, D., Matthäi, S., Finsterle, S., 2007. Electrokinetic coupling in unsaturated porous media. Journal of colloid and interface science 313, 315-327.

Revil, A., Schwaeger, H., Cathles, L., Manhardt, P., 1999. Streaming potential in porous media: 2. theory and application to geothermal systems. Journal of Geophysical Research: Solid Earth 104, 20033-20048.

Rizzo, E., Suski, B., Revil, A., Straface, S., Troisi, S., 2004. Self-potential signals associated with pumping tests experiments. Journal of Geophysical Research: Solid Earth 109, doi:10.1029/2004JB003049.

Saunders, J., Jackson, M., Pain, C., 2006. A new numerical model of electrokinetic potential response during hydrocarbon recovery. Geophysical research letters 33. doi:10.1029/2006GL026835.

Sill, W.R., 1983. Self-potential modeling from primary flows. Geophysics 48, 76-86.

Solazzi, S.G., Guarracino, L., Rubino, J.G., Holliger, K., 2019. Saturation hysteresis effects on the seismic signatures of partially saturated heterogeneous porous rocks. Journal of Geophysical Research: Solid Earth Accepted for publication, https://doi.org/10.1029/2019JB017726.

Soldi, M., Guarracino, L., Jougnot, D., 2017. A simple hysteretic constitutive model for unsaturated flow. Transport in Porous Media 120, 271-285.

Soldi, M., Jougnot, D., Guarracino, L., 2019. An analytical effective excess charge density model to predict the streaming potential generated by unsaturated flow. Geophysical Journal International 216, 380-394.

Soueid Ahmed, A., Jardani, A., Revil, A., Dupont, J.P., 2014. Hydraulic conductivity field characterization from the joint inversion of hydraulic heads and self-potential data. Water Resources Research 50, 3502-3522.

Soueid Ahmed, A., Jardani, A., Revil, A., Dupont, J.P., 2016. Joint inversion of hydraulic head and self-potential data associated with harmonic pumping tests. Water Resources Research 52, 6769-6791.

Stern, O., 1924. Zur theorie der elektrolytischen doppelschicht. Zeitschrift für Elektrochemie und angewandte physikalische Chemie 30, 508-516.

Straface, S., Fallico, C., Troisi, S., Rizzo, E., Revil, A., 2007. An inverse procedure to estimate transmissivity from heads and sp signals. Groundwater 45, 420-428. 
Suski, B., Revil, A., Titov, K., Konosavsky, P., Voltz, M., Dages, C., Huttel, O., 2006. Monitoring of an infiltration experiment using the self-potential method. Water Resources Research 42. doi:10.1029/2005WR004840.

Thanh, L.D., Jougnot, D., Van Do, P., Van Nghia A, N., 2019. A physically based model for the electrical conductivity of water-saturated porous media. Geophysical Journal International 219, 866-876.

Thanh, L.D., Van Do, P., Van Nghia, N., Ca, N.X., 2018. A fractal model for streaming potential coefficient in porous media. Geophysical Prospecting 66, 753-766.

Topp, G., 1971. Soil-water hysteresis: the domain theory extended to pore interaction conditions 1. Soil Science Society of America Journal 35, 219-225.

Topp, G.C., Miller, E., 1966. Hysteretic moisture characteristics and hydraulic conductivities for glass-bead media1. Soil Science Society of America Journal 30, 156-162.

Tyler, S.W., Wheatcraft, S.W., 1990. Fractal processes in soil water retention. Water Resources Research 26, 1047-1054.

Van Genuchten, M.T., 1980. A closed-form equation for predicting the hydraulic conductivity of unsaturated soils. Soil Sci. Soc. Am. J 44, 892-898.

Vogel, H.J., Roth, K., 2001. Quantitative morphology and network representation of soil pore structure. Advances in water resources 24, 233-242.

Voytek, E.B., Barnard, H.R., Jougnot, D., Singha, K., 2019. Transpiration- and precipitation-induced subsurface water flow observed using the self-potential method. Hydrological Processes 33, 1784-1801,https://doi.org/10.1002/hyp.13453.

Wang, S., Wu, T., Qi, H., Zheng, Q., Zheng, Q., 2015. A permeability model for power-law fluids in fractal porous media composed of arbitrary cross-section capillaries. Physica A: Statistical Mechanics and its Applications 437, 12-20, https://doi.org/10.1016/j.physa.2015.05.089.

Waxman, M., Smits, L., 1968. Electrical conductivities in oil-bearing shaly sands. Society of Petroleum Engineers Journal 8, 107-122.

Xu, C., Torres-Verdín, C., 2013. Pore system characterization and petrophysical rock classification using a bimodal gaussian density function. Mathematical Geosciences 45, 753-771.

Yu, B., Li, J., Li, Z., Zou, M., 2003. Permeabilities of unsaturated fractal porous media. International journal of multiphase flow 29, 1625-1642.

Zhang, J., Vinogradov, J., Leinov, E., Jackson, M., 2017. Streaming potential during drainage and imbibition. Journal of Geophysical Research: Solid Earth . 